\title{
Primary Indicator
}

National Cancer Institute

\section{Source}

National Cancer Institute. Primary Indicator. NCI Thesaurus. Code C93631.

Specifies whether this is the main or principal entity. 